\title{
Passive targeting of nanoparticles to cancer: A comprehensive review of the literature
}

\author{
REMON BAZAK ${ }^{1}$, MOHAMAD HOURI ${ }^{2}$, SAMAR EL ACHY $^{3}$, WAEL HUSSEIN $^{1}$ and TAMER REFAAT ${ }^{4,5}$ \\ ${ }^{1}$ Department of Otorhinolaryngology, Faculty of Medicine, Alexandria University, Alexandria 21131, Egypt; \\ ${ }^{2}$ Department of Ophthalmology, Faculty of Medicine, Beirut Arab University, Beirut 1107 2809, Lebanon; \\ Departments of ${ }^{3}$ Pathology, and ${ }^{4}$ Clinical Oncology and Nuclear Medicine, Faculty of Medicine, \\ Alexandria University, Alexandria 21131, Egypt; ${ }^{5}$ Department of Radiation Oncology, \\ Northwestern University, Chicago, IL 60611, USA
}

Received May 23, 2014; Accepted July 2, 2014

DOI: $10.3892 / \mathrm{mco} .2014 .356$

\begin{abstract}
Cancer remains the one of the most common causes of mortality in humans; thus, cancer treatment is currently a major focus of investigation. Researchers worldwide have been searching for the optimal treatment (the 'magic bullet') that will selectively target cancer, without afflicting significant morbidity. Recent advances in cancer nanotechnology have raised exciting opportunities for specific drug delivery by an emerging class of nanotherapeutics that may be targeted to neoplastic cells, thereby offering a major advantage over conventional chemotherapeutic agents. There are two ways by which targeting of nanoparticles may be achieved, namely passive and active targeting. The aim of this study was to provide a comprehensive review of the literature focusing on passive targeting.
\end{abstract}

\section{Contents}

1. Introduction

2. Passive targeting

3. Nanometer size range

4. Abnormal tumor vasculature

5. Factors affecting enhanced permeability and retention

6. Perquisites for enhanced permeability and retention

7. Concluding remarks

Correspondence to: Dr Remon Bazak, Department of Otorhinolaryngology, Faculty of Medicine, Alexandria University, Champollion Street, El-Azareeta, Alexandria 21131, Egypt

E-mail: dr_remon77@yahoo.com

Key words: nanoparticles, cancer, passive targeting

\section{Introduction}

Cancer remains the one of the most common causes of mortality in humans; thus, cancer treatment is currently a major focus of investigation (1). Intense efforts aimed at improving conventional treatment did not achieve the desired goal and there has been little advancement on the overall cancer survival landscape. Chemotherapeutics used in cancer management have one prime purpose, which is eliminating malignant cells, a task usually achieved with high efficacy but little precision (2). Patients subjected to treatment paradigms with such non-specific toxic compounds commonly develop severe side effects that may be debilitating in their own right $(3,4)$. Ehrlich introduced the concept of the 'magic bullet' at the turn of the 20 th century (5). Since then, researchers worldwide have been searching for an optimal treatment that selectively targets cancer without afflicting significant morbidity. Recent advances in cancer nanotechnology have raised exciting opportunities for specific drug delivery by an emerging class of nanotherapeutics that may be targeted to neoplastic cells, thereby offering a major advantage over conventional chemotherapeutic agents $(4,6)$. There are two ways by which targeting of nanoparticles may be achieved, namely passive and active targeting.

Passive targeting facilitates deposition of nanovectors within the tumor microenvironment, owing to distinctive characteristics inherent to the tumor milieu, not normally present in healthy tissues (5). The delivery of nanoparticles is determined by factors associated with the tumor microvasculature, in addition to factors inherent to the nanoparticle itself, such as size, shape and surface charge (5). Targeting strategies have taken a step further to enhance the selective uptake of nanoparticles into the tumor cells. Biorecognition molecules have been attached to the surface of the nanovectors to target specific markers that are overexpressed by the neoplastic cells. These strategies have been awarded the appellation 'active targeting', which exhibits a higher specificity and efficacy in achieving the desired goal (6). The aim of this study was to provide an overview of the factors orchestrated in the passive targeting of nanoparticles to tumors. In deference to the traditional views, we also aimed to investigate the various strategies that may be adopted to maximize the benefits of this approach. 


\section{Passive targeting}

In passive targeting, macromolecules including nanoparticles accumulate preferentially in the neoplastic tissues as a result of the enhanced permeability and retention (EPR) phenomenon, first described by Maeda and Matsumura $(7,8)$. The EPR is based on the nanometer size range of the nanoparticles and two fundamental characteristics of the neoplastic tissues, namely, the leaky vasculature and impaired lymphatic drainage.

\section{Nanometer size range}

In 1986, Maeda et al (9) observed that intravenous administration of Evans blue dye, which binds to plasma albumin, resulted in selective concentration in tumor tissues. The tumor concentration of blue albumin mounted to $\sim 10$-fold that in the blood at $145 \mathrm{~h}$. This phenomenon was also demonstrated with radio-labeled plasma proteins, including transferrin $(90 \mathrm{kDa})$ and IgG $(160 \mathrm{kDa})$, whereas smaller proteins, such as neocarzinostatin (12 kDa) and ovomucoid (29 kDa), did not accumulate within tumors $(1,8,10)$. Subsequent studies have confirmed that macromolecules with a molecular weight above the renal threshold $(40 \mathrm{kDa})$ tend to accumulate preferentially in neoplastic tissues upon intravenous administration $(1,11)$. This unique phenomenon of preferential accumulation of macromolecules is the resultant effect of the abnormal vasculature and impaired lymphatic drainage within neoplastic tissues.

\section{Abnormal tumor vasculature}

Once a malignant tumor grows to $>2-3 \mathrm{~mm}^{3}$ in size, the delivery of oxygen and nutrients becomes diffusion-limited and the formation of new blood vessels becomes essential to meet the ever increasing demands of the rapidly growing malignant cells (3). This is accomplished through the release of angiogenic factors by the neoplastic tissue aiming to increase the microvasculature within the tumor in order to sustain further growth (3). The resultant imbalance of angiogenic factors and matrix metalloproteinases (MMPs) within neoplastic tissues results in highly disorganized vessels, which are dilated, with numerous pores and wide gap junctions between endothelial cells (12). The perivascular cells and basement membrane are absent or defective $(1,13)$. Furthermore, tumor vessels frequently lack the smooth muscle layer that normally surrounds endothelial cells (14). The normal vasculature is endowed with tight junctions that are impermeable to molecules sized $>2-4 \mathrm{~nm}$, thus keeping the nanoparticles within the circulation; however, the leaky vasculature of neoplastic tissue allows macromolecules with a diameter of $\geq 600 \mathrm{~nm}$ to extravasate into the neoplastic tissues. Since tumors do not have a well-developed lymphatic system, these extravasated nanoparticles tend to stagnate within the neoplastic tissue $(12,15)$. This phenomenon of leaky vasculature and impaired lymphatic drainage has been referred to as the EPR effect $(7,8)$.

\section{Factors affecting enhanced permeability and retention}

Architectural abnormalities of the neoplastic vessels and blood pressure. In normal blood vessels, the smooth muscle layer is essential for mediating a vasogenic response to vascular mediators and, hence, for maintaining a constant blood flow to an organ. By contrast, the microvasculature in neoplastic tissues lacks these smooth muscle cells; therefore, these vessels are in a state of permanent vasodilation and non-responsiveness to physiological stimuli regulating blood flow (16). These aberrant neoplastic vessels result in abnormal transport dynamics of fluid and solutes across tumor vessels, which may be exploited to further accentuate the EPR effect (1).

Suzuki et al (17) demonstrated that elevating the mean arterial blood pressure by infusion of angiotensin II resulted in an 5.7-fold selective increase in blood flow in tumor tissue, without an associated increase in normal tissue. Li et al (18) later verified that angiotensin II-induced hypertension augments the EPR effect. Raising the systolic blood pressure in tumor-bearing rats by angiotensin II infusion resulted in a 2-6-fold selective increase in tumor blood flow volume, depending on the blood pressure attained. In addition to the increased blood flow, the authors of that study observed a preferential accumulation of drugs with a molecular mass of $\sim 80 \mathrm{kDa}$ within the tumor tissue. Moreover, drug accumulation in normal organs, such as kidney and bone marrow, was reduced to $60-80 \%$. Tight endothelial gap junctions and normal vasogenic response to angiotensin in healthy tissues permit less transvascular transfer of macromolecules. By contrast, lack of a vasogenic response due to the deficient vascular smooth muscle layer in the neoplastic blood vessels results in an increased intratumoral blood flow in responose to a systemic elevation of the blood pressure. An increased blood flow and a leaky vasculature result in accumulation of macromolecular drugs in neoplastic tissues. Similar results have been observed upon systemic administration of macromolecular drugs to patients with several solid tumors under an angiotensin II-induced hypertensive state $(13,19)$. While low-molecular weight anticancer drugs have a dose-limiting toxicity, using macromolecular agents under a hypertensive state achieved a $>5$-fold higher concentration of anticancer drugs in the tumor, even though the hypertension was maintained only for $\sim 20 \min (13,19)$.

Vasogenic mediators. The tumor microvasculature is orchestrated by a number of local mediators, including bradykinin, nitric oxide (NO), peroxynitrite, MMPs, vascular endothelial growth factor (VEGF) and prostaglandins (PGs). These mediators have been investigated in an attempt to potentiate the EPR and, hence, achieve better drug targeting to the neoplastic tissue (Table I) (20).

Bradykinin. The Hageman factor (factor XII) of the coagulation cascade is the chief protease of the kallikrein-kinin system. The activation of factor XII is followed by activation of prekallikrein to kallikrein. Kallikrein generates bradykinin directly from kininogen (21). Bradykinin receptors have been identified in various human and rodent solid tumors $(22,23)$ and it was demonstrated that the bradykinin-generating cascade is activated in neoplastic tissues (24). Bradykinin is present at high levels in the peritoneal and pleural fluids of humans and animals with cancer. The crucial role of bradykinin in the extravasation of plasma components into the peritoneal or pleural cavity was further demonstrated by the inhibition of kallikrein (19). Therefore, bradykinin, a key factor controlling 
Table I. A summary of the effects of different mediators on the microvasculature of solid tumors.

\begin{tabular}{|c|c|c|}
\hline Mediator & Effect & (Refs.) \\
\hline Angiotensin II & $\begin{array}{l}\text { Raises the systolic blood pressure, thereby increasing blood flow } \\
\text { Lack of normal vasogenic response to angiotensin increases } \\
\text { the permeability of tumor vessels }\end{array}$ & $(27-31)$ \\
\hline Bradykinin & $\begin{array}{l}\text { Controls vascular permeability } \\
\text { Activates NO production through eNOS }\end{array}$ & $\begin{array}{l}(13,37) \\
(40,41)\end{array}$ \\
\hline ACE inhibitors (e.g., enalapril) & $\begin{array}{l}\text { Enhance vascular permeability effects of bradykinin } \\
\text { ACE inhibitors increase delivery of macromolecular drugs to tumors, } \\
\text { even under normotensive conditions }\end{array}$ & $\begin{array}{c}(33) \\
(3,33,45,46)\end{array}$ \\
\hline Nitric oxide & $\begin{array}{l}\text { Induces dilation of tumor vessels } \\
\text { Promotes angiogenesis } \\
\text { Increases vascular permeability } \\
\text { Indirect activation of MMPs }\end{array}$ & $(20,33)$ \\
\hline Peroxynitrite and MMPs & $\begin{array}{l}\text { Degrade ECM and enhance angiogenesis and metastasis } \\
\text { Enhance vascular permeability }\end{array}$ & $\begin{array}{c}(58,59) \\
(20,32,60)\end{array}$ \\
\hline Vascular endothelial growth factor & Up to 30 -fold increase in vascular permeability in neoplastic tissues & $(33,47)$ \\
\hline Prostaglandins & $\begin{array}{l}\text { PGE2 and PGI2 enhance vascular permeability } \\
\text { PGI2 analogues enhance the EPR effect in tumors up to } 3 \text {-fold }\end{array}$ & $\begin{array}{l}(62,63) \\
(64)\end{array}$ \\
\hline
\end{tabular}

NO, nitric oxide; eNOS, endothelial nitric oxide synthase; ACE, angiotensin-converting enzyme; MMPs, matrix metalloproteinases; ECM, extracellular matrix; PG, prostaglandin; EPR, enhanced permeability and retention.

vascular permeability, is an important mediator controlling the EPR effect in neoplastic tissues $(11,24)$. Bradykinin is also known to activate NO production via the activation of endothelial NO synthase (eNOS) (25). NO production contributes to the angiogenic properties of VEGF in human endothelial cells (26), also referred to as vascular permeability factor (VPF) (27).

Bradykinin is degraded by several peptidases, particularly angiotensin-converting enzyme (ACE) (21). Inhibition of ACE is expected to increase the local concentration of bradykinin and, hence, increase the vascular permeability of the tumor. It was reported that ACE inhibitors, such as enalapril and temocapril, potentiate the EPR effect $(28,29)$. More importantly, ACE inhibitors increase the delivery of macromolecular drugs to tumors, even under normotensive conditions (2). ACE inhibitors are non-toxic, without major adverse effects in healthy individuals and are only active in hypertensive patients. Therefore, ACE inhibitors may act selectively at tumor sites in normotensive patients with neoplasia to potentiate the EPR effect (11).

$N O$. NO is generated from $\mathrm{L}$-arginine and oxygen by three isoforms of NOS. Inducible NOS, the most potent isoform, is produced in macrophages and neutrophils, which are known to extensively infiltrate tumor tissues $(11,21,30)$. $\mathrm{NO}$ is a well-known mediator of vasodilation, angiogenesis and extravasation $(13,21)$. It has been demonstrated that enhanced vascular permeability in solid tumors is mediated by NO and inhibited by NO scavengers and NO synthase inhibitors $(19,31)$. As a mediator affecting tumor vascular permeability, $\mathrm{NO}$ is expected to play a critical role in enhancing the EPR effect in solid tumors (21). Apart from exerting a direct effect on EPR, NO reacts rapidly with superoxide anion, which is predominantly produced by leukocytes, to generate peroxynitrite. The formed peroxynitrite, in turn, activates MMP precursors (proMMPs) into MMPs (32), which may also contribute to the EPR effect $(21,32)$.

When the NO-releasing agent isosorbide dinitrate was infused into the local tumor feeding artery and angiotensin II was concomitantly injected systemically, the site-specific delivery of SMANCS-Lipiodol was enhanced, supporting the hypothesis that NO enhances the EPR effect (13). An analogy between hypoxic solid tumor and ischemic cardiac tissue in angina pectoris has been described (33). Nitroglycerin used in the management of angina pectoris liberates nitrite through the action of denitrase, which is then converted to NO in the ischemic tissues by nitrite reductase $(11,33)$. This pharmacological benefit of nitroglycerin has also been validated in vivo, in a mouse tumor model (34). Topical application of nitroglycerin ointment to the skin of mice with breast cancer resulted in an increased blood flow only in the neoplastic tissue, thereby increasing macromolecular drug delivery to the tumor. Clinical evaluations of nitroglycerin used in combination with conventional low-molecular weight anticancer agents were recently undertaken by Yasuda et al $(35,36)$ and Siemens et al (37); both studies reported significant clinical improvement in therapeutic response, indicating that NO clearly benefits patients undergoing chemotherapy (11).

Peroxynitrite and MMPs. Neoplastic tissues synthesize NO, which reacts with superoxide anion generated by the recruited inflammatory cells, generating peroxynitrite (30). ProMMPs react with peroxynitrite and are activated to MMPs (13). MMPs are known to facilitate cancer metastasis by degrading the extracellular matrix and to enhance angiogenesis, thus 
supporting the growth of solid tumors $(38,39)$. It has been demonstrated that MMPs also enhance the vascular permeability of solid tumors in mice and this effect was shown to be suppressed by MMP inhibitors $(13,20,40)$. Several MMP inhibitors have been developed over the last two decades; however, none of these inhibitors was applicable clinically. The first reason for this failure may be that some tumor cells remain viable and, therefore, may resume growth when the drug treatment is discontinued. The second reason may be that MMPs are proteases vital for cellular metabolism and high doses of MMP inhibitors cause toxicity. This has led to the termination of the development of a number of anti-MMP drugs (13).

$V E G F$. VEGF, formerly known as VPF, has been shown to be 2- to 30-fold higher in neoplastic compared to normal tissues, with the exception of the lung $(21,30)$. In addition to being a mitogen for endothelial cells, VEGF plays a pivotal role in the induction of vascular permeability (13). Intradermal injection of VEGF has been shown to significantly enhance the extravasation of Evans blue dye in a dose-dependent manner, thereby highlighting its important role in enhancing the EPR effect $(21,30)$.

PGs. PGs, particularly PGE2, are important mediators of vascular permeability. PGE2 is generated via cyclooxygenase (COX) isozymes, such as COX-2, which is markedly elevated in tumors. The suppression of vascular permeability in sarcoma 180 and other solid tumors by COX inhibitors, such as indomethacin and salicylic acid, provides solid evidence for the role of PGs in enhancing vascular permeability $(1,19)$. A PGI2 analogue, beraprost sodium, exhibits a significantly longer half-life in vivo ( $>1 \mathrm{~h}$ ) compared to PGI2, which only lasts for a few seconds. Tanaka et al (41) demonstrated that PGI2 analogues may enhance the EPR effect by 2- to 3 -fold, thereby providing a useful strategy for macromolecule delivery.

\section{Perquisites for enhanced permeability and retention}

Nanoparticles may attain high concentrations within the neoplastic tissue via the EPR effect only if they are able to evade the reticuloendothelial system (RES) and resist renal clearance by virtue of their macromolecular size, thereby remaining in the circulation for $\geq 6 \mathrm{~h}$ (1).

Evasion of the RES. Upon intravenous administration, nanoparticles are rapidly recognized as foreign particles and are opsonized by the adsorption of plasma proteins. The opsonized nanoparticles interact with specific receptors on Kupffer cells in the liver and macrophages in the spleen and are thus rapidly eliminated from the systemic circulation (42). The rapid clearance of the nanoparticles from the systemic circulation by the RES results in a short circulation half-life, which is not adequate to permit the accumulation of the nanoparticles within the neoplastic tissues $(15,42,43)$. In order to prolong the half-life of nanoparticles in the circulation, they have to be converted to stealth nanoparticles, thereby evading opsonization and uptake by the RES cells. Evasion of opsonization is based on the physicochemical concept of steric repulsion, by grafting polyethylene glycol (PEG) residues or polysaccharides onto the nanoparticle surface (43). The presence of such macromolecules creates a 'steric stabi- lization', which provides a protective hydrophilic layer on the surface of nanoparticles, preventing aggregation between the particles themselves, as well as their interaction with blood components (44). This masking effect confers nanoparticles with the ability to evade the cells of the RES $(45,46)$. PEG is the most widely used material for surface modification, as it is non-toxic, non-immunogenic and has been approved by the United States Food and Drug Administration for oral and parenteral applications in humans (43). PEGylated nanoparticles exhibit a circulation half-life of 2-24 h in mice and rats and as long as $45 \mathrm{~h}$ in humans, thus providing the nanoparticles with sufficient time to reach their target tissue (45).

Macromolecular size. The EPR effect is a molecular weight-dependent phenomenon. Molecules exhibiting a molecular weight below the renal clearance threshold are rapidly eliminated from the circulation. However, a drug has to remain in the circulation for $\geq 6 \mathrm{~h}$ to be able to accumulate in the neoplastic tissue by the EPR effect (20). Therefore, the EPR effect is particular to macromolecules with an apparent molecular size of $>40-50 \mathrm{kDa}(20,47)$.

\section{Concluding remarks}

With the rapid emergence of novel nanoparticulate devices, there comes a pressing need for greater precision in delivering drugs to neoplastic cancer cells, whilst salvaging the surrounding healthy tissues. The tumor microvasculature represents the epicenter of the concept of passive targeting of nanoparticles. Mediators regulating blood pressure and vascular caliber may be controlled to shift the balance towards a more inviting tumor environment for nanoparticles. Skeptics point to the fact that passive targeting facilitates the efficient localization of nanoparticles in the tumor interstitium, but cannot further promote their uptake by cancer cells. This uptake may be achieved by actively targeting nanoparticles to receptors overexpressed on target cancer cells. It is our view that the two strategies must be synchronized in order to achieve maximum benefit from future nanodesigned 'magic bullets'.

\section{References}

1. Greish K: Enhanced permeability and retention of macromolecular drugs in solid tumors: a royal gate for targeted anticancer nanomedicines. J Drug Target 15: 457-464, 2007.

2. Greish K: Enhanced permeability and retention (EPR) effect for anticancer nanomedicine drug targeting. Methods Mol Biol 624: 25-37, 2010.

3. LaRocque J, Bharali DJ and Mousa SA: Cancer detection and treatment: the role of nanomedicines. Mol Biotechnol 42: 358-366, 2009.

4. Wang X, Wang Y, Chen ZG and Shin DM: Advances of cancer therapy by nanotechnology. Cancer Res Treat 41: 1-11, 2009.

5. Kreuter J: Nanoparticles - a historical perspective. Int J Pharm 331: 1-10, 2007.

6. Byrne JD, Betancourt T and Brannon-Peppas L: Active targeting schemes for nanoparticle systems in cancer therapeutics. Adv Drug Deliv Rev 60: 1615-1626, 2008.

7. Maeda $\mathrm{H}$ and Matsumura Y: Tumoritropic and lymphotropic principles of macromolecular drugs. Crit Rev Ther Drug Carrier Syst 6: 193-210, 1989.

8. Matsumura Y and Maeda H: A new concept for macromolecular therapeutics in cancer chemotherapy: mechanism of tumoritropic accumulation of proteins and the antitumor agent smancs. Cancer Res 46: 6387-6392, 1986. 
9. Maeda H, Matsumura Y and Oda T: Cancer selective macromolecular therapeutics: tailoring of an antitumor protein drug. In: Protein Tailoring for Food and Medical Uses. Feeny RE and Whitaker JR (eds). Marcel Dekker, Inc., New York, pp353-382, 1986.

10. Maeda H, Takeshita J and Kanamaru R: A lipophilic derivative of neocarzinostatin. A polymer conjugation of an antitumor protein antibiotic. Int J Pept Protein Res 14: 81-87, 1979.

11. Maeda H: Vascular permeability in cancer and infection as related to macromolecular drug delivery, with emphasis on the EPR effect for tumor-selective drug targeting. Proc Jpn Acad Ser B Phys Biol Sci 88: 53-71, 2012.

12. Cho K, Wang X, Nie S, Chen ZG and Shin DM: Therapeutic nanoparticles for drug delivery in cancer. Clin Cancer Res 14: 1310-1316, 2008.

13. Iyer AK, Khaled $\mathrm{G}$, Fang $\mathbf{J}$ and Maeda $\mathrm{H}$ : Exploiting the enhanced permeability and retention effect for tumor targeting. Drug Discov Today 11: 812-818, 2006.

14. Skinner SA, Tutton PJ and O'Brien PE: Microvascular architecture of experimental colon tumors in the rat. Cancer Res 50 2411-2417, 1990

15. Talekar M, Kendall J, Denny W and Garg S: Targeting of nanoparticles in cancer: drug delivery and diagnostics. Anticancer Drugs 22: 949-962, 2011.

16. Kuruppu D, Christophi C, Maeda H and O'Brien PE: Changes in the microvascular architecture of colorectal liver metastases following the administration of SMANCS/lipiodol. J Surg Res 103: 47-54, 2002

17. Suzuki M, Hori K, Abe I, Saito S and Sato H: A new approach to cancer chemotherapy: selective enhancement of tumor blood flow with angiotensin II. J Natl Cancer Inst 67: 663-669, 1981.

18. Li CJ, Miyamoto Y, Kojima Y and Maeda H: Augmentation of tumour delivery of macromolecular drugs with reduced bone marrow delivery by elevating blood pressure. Br J Cancer 67 975-980, 1993

19. Wu J, Akaike T and Maeda H: Modulation of enhanced vascular permeability in tumors by a bradykinin antagonist, a cyclooxygenase inhibitor, and a nitric oxide scavenger. Cancer Res 58: $159-165,1998$

20. Maeda H: The enhanced permeability and retention (EPR) effect in tumor vasculature: the key role of tumor-selective macromolecular drug targeting. Adv Enzyme Regul 41: 189-207, 2001.

21. Seki T, Fang J and Maeda H: Tumor-targeted macromolecular drug delivery based on the enhanced permeability and retention effect in solid tumor. In: Pharmaceutical Perspectives of Cancer Therapeutics. Lu Y and Mahato RI (eds). Springer, New York, pp93-120, 2009.

22. Wu J, Akaike T, Hayashida K, Miyamoto Y, Nakagawa T, Miyakawa K, Müller-Esterl W and Maeda H: Identification of bradykinin receptors in clinical cancer specimens and murine tumor tissues. Int J Cancer 98: 29-35, 2002.

23. Bhoola K, Ramsaroop R, Plendl J, Cassim B, Dlamini Z and Naicker S: Kallikrein and kinin receptor expression in inflammation and cancer. Biol Chem 382: 77-89, 2001.

24. Matsumura Y, Kimura M, Yamamoto $T$ and Maeda $H$ Involvement of the kinin-generating cascade in enhanced vascular permeability in tumor tissue. Jpn J Cancer Res 79: 1327-1334, 1988

25. Kou R, Greif D and Michel T: Dephosphorylation of endothelial nitric-oxide synthase by vascular endothelial growth factor. Implications for the vascular responses to cyclosporin A. J Biol Chem 277: 29669-29673, 2002.

26. Papapetropoulos A, Garcia-Cardena G, Madri JA and Sessa WC Nitric oxide production contributes to the angiogenic properties of vascular endothelial growth factor in human endothelial cells. J Clin Invest 100: 3131-3139, 1997.

27. Maeda H, Wu J, Okamoto T, et al: Kallikrein-kinin in infection and cancer. Immunopharmacology 43: 115-128, 1999.

28. Hori K, Saito S, Takahashi H, Sato H, Maeda H and Sato Y: Tumor-selective blood flow decrease induced by an angiotensin converting enzyme inhibitor, temocapril hydrochloride. Jpn J Cancer Res 91: 261-269, 2000 .
29. Noguchi A, Takahashi T, Yamaguchi T, et al: Enhanced tumor localization of monoclonal antibody by treatment with kininase II inhibitor and angiotensin II. Jpn J Cancer Res 83: 240-243, 1992.

30. Maeda H, Fang J, Inutsuka T and Kitamoto Y: Vascular permeability enhancement in solid tumor: various factors, mechanisms involved and its implications. Int Immunopharmacol 3: 319-328, 2003.

31. Maeda H, Noguchi Y, Sato K and Akaike T: Enhanced vascular permeability in solid tumor is mediated by nitric oxide and inhibited by both new nitric oxide scavenger and nitric oxide synthase inhibitor. Jpn J Cancer Res 85: 331-334, 1994.

32. Okamoto T, Akaike T, Sawa T, Miyamoto Y, van der Vliet A and Maeda $\mathrm{H}$ : Activation of matrix metalloproteinases by peroxynitrite-induced protein S-glutathiolation via disulfide S-oxide formation. J Biol Chem 276: 29596-29602, 2001.

33. Maeda H: Nitroglycerin enhances vascular blood flow and drug delivery in hypoxic tumor tissues: analogy between angina pectoris and solid tumors and enhancement of the EPR effect. J Control Release 142: 296-298, 2010.

34. Seki T, Fang J and Maeda H: Enhanced delivery of macromolecular antitumor drugs to tumors by nitroglycerin application. Cancer Sci 100: 2426-2430, 2009.

35. Yasuda H, Yamaya M, Nakayama K, et al: Randomized phase II trial comparing nitroglycerin plus vinorelbine and cisplatin with vinorelbine and cisplatin alone in previously untreated stage IIIB/IV non-small-cell lung cancer. J Clin Oncol 24: 688-694, 2006

36. Yasuda H, Nakayama K, Watanabe M, et al: Nitroglycerin treatment may enhance chemosensitivity to docetaxel and carboplatin in patients with lung adenocarcinoma. Clin Cancer Res 12: 6748-6757, 2006

37. Siemens DR, Heaton JP, Adams MA, Kawakami J and Graham CH: Phase II study of nitric oxide donor for men with increasing prostate-specific antigen level after surgery or radiotherapy for prostate cancer. Urology 74: 878-883, 2009.

38. Himelstein BP, Canete-Soler R, Bernhard EJ, Dilks DW and Muschel RJ: Metalloproteinases in tumor progression: the contribution of MMP-9. Invasion Metastasis 14: 246-258, 1994.

39. Chambers AF and Matrisian LM: Changing views of the role of matrix metalloproteinases in metastasis. J Natl Cancer Inst 89: 1260-1270, 1997

40. Wu J, Akaike T, Hayashida K, Okamoto T, Okuyama A and Maeda $\mathrm{H}$ : Enhanced vascular permeability in solid tumor involving peroxynitrite and matrix metalloproteinases. Jpn J Cancer Res 92: 439-451, 2001.

41. Tanaka S, Akaike T, Wu J, Fang J, Sawa T, Ogawa M, et al: Modulation of tumor-selective vascular blood flow and extravasation by the stable prostaglandin 12 analogue beraprost sodium. J Drug Target 11: 45-52, 2003.

42. Moghimi SM, Hunter AC and Murray JC: Long-circulating and target-specific nanoparticles: theory to practice. Pharmacol Rev 53: 283-318, 2001.

43. Huynh NT, Roger E, Lautram N, Benoit JP and Passirani C: The rise and rise of stealth nanocarriers for cancer therapy: passive versus active targeting. Nanomedicine (Lond) 5: 1415-1433, 2010

44. Wang $M$ and Thanou M: Targeting nanoparticles to cancer. Pharmacol Res 62: 90-99, 2010.

45. Singh R and Lillard JW Jr: Nanoparticle-based targeted drug delivery. Exp Mol Pathol 86: 215-223, 2009.

46. Torchilin VP: Passive and active drug targeting: drug delivery to tumors as an example. Handb Exp Pharmacol 197: 3-53, 2010.

47. Fang J, Nakamura $\mathrm{H}$ and Maeda H: The EPR effect: unique features of tumor blood vessels for drug delivery, factors involved, and limitations and augmentation of the effect. Adv Drug Deliv Rev 63: 136-151, 2011. 